\title{
El reino desplegado. La mirada oblicua en la trilogía fronteriza de Yuri Herrera*
}

\author{
Luis Enrique Escamilla Frías ${ }^{1}$ \\ Universidad Iberoamericana (Ciudad de México)
}

\section{Resumen}

Si se considera la trilogía fronteriza de Yuri Herrera solo por los grandes temas con que se relaciona, tendríamos que Trabajos del reino (2004) se refiere al narcotráfico de los últimos años en México, Señales que precederán al fin del mundo (2009) a la migración México-EEUU y La transmigración de los cuerpos (2013) a la violencia y el miedo social presentes en Latinoamérica. Lo interesante es que las tres se aproximan a esos tópicos de forma oblicua. De allí que, para analizar tanto la posición lateral de las novelas como su eventual reinterpretación sobre esos temas, en esta investigación se trabaja a partir de Jacques Lacan -en particular, su concepto de anamorfosis (mirar oblicuamente) y su esquema de los órdenes Real, Imaginario y Simbólico. Y se llega al siguiente resultado: mientras las primeras dos novelas sí logran un trastrocamiento de los discursos consabidos sobre el narcotráfico y la migración, la tercera no lo logra respecto a la violencia.

Palabras clave: Latinoamérica, discursos de poder, mirada oblicua, anamorfosis, resimbolización.

\section{Abstract}

If we consider the Yuri Herrera's border trilogy just because it relates great issues, Kingdom Cons (2004) refers to the drug trafficking of recent years in Mexico, Signs Preceding the End of the World (2009) to the Mexico-US migration, and The transmigration of bodies (2013) to violence and social fear present in Latin America. The interesting thing is that the three approach these topics obliquely. So to analyze both the lateral position of the novels and their eventual reinterpretation of these themes, this research is based on, among other authors, Jacques Lacan -in particular, his concept of anamorphosis (looking obliquely) and his scheme of the Real, Imaginary and Symbolic orders. And this result is reached in the following pages: while the first two novels do achieve a reversal of the usual ideas about drug trafficking and migration, the third does not achieve so with respect to violence.

Keywords: Latin America, power discourse, oblique looking, anamorphosis, re symbolization.

\footnotetext{
* The unfolded kingdom. The oblique looking in the Yuri Herrera's border trilogy 1 Magíster en Letras Modernas por la Universidad Iberoamericana, Ciudad de México. Correo electrónico: luis.enrique.frias@gmail.com
} 


\section{Introducción}

La primera novela de la trilogía fronteriza, Trabajos del reino (2004), es la tesis con que Yuri Herrera (México, 1970) se graduó de la maestría en Creación Literaria de la Universidad de Texas, en El Paso. Cuenta la historia de Lobo, un adolescente paupérrimo, cantante de narcocorridos, que anda de cantina en cantina sin encontrarse a sí mismo, hasta que un día conoce en una de ellas al Rey, cabeza de un cártel: el Reino. Lobo entra en este ámbito, y desde una posición lateral, oblicua, comprenderá algunos de los entresijos del narcotráfico. Pero en la novela nunca se mencionan términos como narcotraficante, narcocorrido, frontera México-EEUU, droga, etc. A lo largo de 26 capítulos breves, sin nombre ni numeración, se cuenta la historia de Lobo con un tempo narrativo predominantemente de escena. Cada capítulo - salvo aquellos donde hay un claro despliegue de la voz narrativa por mostrar los pensamientos de Lobo- se presenta con esa velocidad, lo que les da la apariencia de ser apenas unos cuantos momentos seleccionados de una historia aún más extensa.

Al igual que en las otras dos novelas de la trilogía fronteriza, Señales que precederán al fin del mundo (2009) y La transmigración de los cuerpos (2013), Herrera parece abordar los temas de frente; pero en un segundo análisis se aprecia que, por el contrario, los temas son otros, en absoluto más sencillos, como el funcionamiento del narco. Pero están allá atrás, algo similar a un trasfondo cinematográfico.

La siguiente novela, Señales que precederán al fin del mundo, escrita por Herrera casi al mismo tiempo que hacía el doctorado en Lengua y Literatura Hispánicas en la Universidad de California, en Berkeley, presenta la historia de Makina, una muchacha que domina tres idiomas: lengua, lengua latina y lengua gabacha ${ }^{2}$. Ella tuvo que emprender un viaje a solicitud de su madre, la Cora, quien le había pedido llevar un recado a su hermano, que se había ido hacía tiempo al gabacho a reclamar una tierra que supuestamente le habría pertenecido a su padre. Makina se vio impelida a dejar la paupérrima Ciudadcita, donde pasaba sus días operando una 'centralita' telefónica, haciendo uso de las tres lenguas que dominaba. Emprende así el viaje al Gran Chilango, donde toma el autobús que la lleva hacia el norte. Una vez allí, Makina cruza un río, y se va adentrando cada vez más en el gabacho. A lo largo de 9 capítulos - mismo número de las escalas que según el mito náhuatl habían de emprender los antiguos mexicas en su descenso al Mictlán-, Makina entra a una sociedad donde sus tres lenguas le ayudan a descifrar lo que desde su posición oblicua des-

2 Lo que la novela nombra como "lengua gabacha" se puede entender como idioma inglés, dado que en México se le denomina popularmente "gabacho" a lo que tenga relación con EE UU. 
cubre con la mirada: una forma diferente de hablar y de comportarse de sus paisanos, colores de piel muy distintos a los de la Ciudadcita, un habla que al mezclar las lenguas gabacha y latina, parece el reflejo de su propia condición cambiante. Casi al término de la novela, Makina encuentra a su hermano, quien también es muy diferente. Se ha transformado prácticamente en un ciudadano de ese país, luego de haberse enrolado en las filas del ejército. Por último, Makina desciende a una cámara oscura "donde no hay ventanas, ni orificios para el humo" (Herrera, 2011: 113), muy parecida a la novena cámara que envolvía al final a los antiguos mexicas en una oscuridad absolutamente silenciosa, de acuerdo con el mito del Mictlán.

La última novela de la trilogía fronteriza es La transmigración de los cuerpos. Alfaqueque, el protagonista, se ocupa del verbo, es decir, posee el don del convencimiento y la intermediación para solucionar conflictos entre pandillas rivales, familias en problemas, etc. "Verbo y verga, es lo único que tengo", reflexiona en un punto el Alfaqueque (Herrera, 2013: 133). La historia comienza cuando el Delfín le llama para pedirle ayuda en un intercambio, que, poco a poco, nos enteramos, era de dos cadáveres humanos. Uno está en manos de la familia Castro, y el otro en poder de los Fonseca. Al Alfaqueque le corresponderá investigar qué pasó realmente: por qué razón los Castro se llevaron el cuerpo de Romeo, y luego cómo se dieron las cosas para que los Fonseca tomaran presa a La Muñe, y así, finalmente, procurar un intercambio pacífico de los cuerpos.

El material crítico que predomina sobre la novela ha destacado el guiño a la crisis de influenza AH1N1 que asoló a México entre 2009 y 2010. Más aún, se ha puesto atención en un elemento decisivo en la historia: el miedo. Al respecto, Herrera ha sostenido que "pensaba en esa epidemia no declarada que es el miedo que nos tenemos los unos a los otros, el recelo, el odio. Esta es, en términos poéticos, la epidemia de nuestro siglo" (Rodríguez Court, 2013: parr. 20).

Las temáticas, la poética y el estilo de las tres novelas las hace próximas entre sí y susceptibles de ser agrupadas bajo una trilogía. Herrera mismo ha dicho, a propósito de La transmigración de los cuerpos, que bien puede asumirse como una novela en border condition, esto es, "the exchange of goods and symbolic values, the creation of new identities, of new linguistic forms, of new political practices"3 (Herrera, 2016, parr. 3). Las tres novelas se agrupan bajo esta forma de asimilar lo fronterizo. Las tres comparten semejanzas entre tensiones territoriales, de lenguaje, de adaptación al entorno, de identidades, con ciertas semejanzas, lo cual me permite proponer que en las tres

3 "El intercambio de bienes y valores simbólicos, la creación de nuevas identidades, de nuevas formas lingüísticas, de nuevas prácticas políticas” (trad. mía). 
se establece un diálogo enriquecedor y se observa una trilogía, que he llamado "la trilogía fronteriza".

Entre otras aproximaciones, desde las cuales he partido para esta investigación, se encuentra la de Ivonne Sánchez Becerril (2014), quien analiza también la marginalidad en la obra herreriana, proponiendo como eje la mirada. Afirma que las novelas parecen mirar los grandes temas que las atraviesan de manera lateral, y no centralmente. Sostiene que, por ejemplo, "el análisis de la mirada de soslayo que hace Señales que precederán al fin del mundo de la movilidad internacional pone en relieve la complejidad e impacto del fenómeno de largo plazo en la transformación social tanto de México como de Estados Unidos" (Herrera, 2009: 108).

En efecto, además de la alegoría antes mencionada, que aúna migración y el mito mexica del Mictlán, están presentes varios temas, debido, entre otras razones, a la posibilidad que brinda el recurso de mirarlos de soslayo.

Si bien Sánchez Becerril habla de una novela particular, lo cierto es que la operación que describe puede abarcar toda la trilogía: gracias a la operación de mirar de soslayo, las novelas nos facultan para ver hacia otros fenómenos que se encuentran en el trasfondo de las historias principales, y en ese acto de mirarlos de lado, los pueden trastocar. Esto es, las novelas nos permiten mirar hacia determinados temas de manera sesgada; y al verlos así, de un modo distinto, las concepciones que teníamos sobre ellos se transforman, lo cual redunda en que los entendamos de modos novedosos.

Por esta razón, el marco teórico que planteo se basa en esa operación de mirar oblicuamente. Slavoj Žižek, en un comentario sobre la película Children of men (Dir. Alfonso Cuarón, EUA-U.K., 2006), pone sobre la mesa el funcionamiento de la mirada lateral. Es una forma de interpretación que puede funcionar en sí misma como una metodología para leer dispositivos artísticos con vocación de mirada oblicua. Exactamente en ese sentido lo hago para el caso de la trilogía fronteriza, que aborda grandes temas contemporáneos, pero desde los márgenes.

El planteamiento central de este artículo es analizar la apuesta de la trilogía fronteriza para plantear desde la mirada oblicua determinados órdenes de lo Simbólico, el cual entiendo, con base en Jacques Lacan, como esa especie de gran estructura conceptual en la que se sostienen los imaginarios que los sujetos tienen sobre lo Real (abundaré sobre esto en el apartado Construcción de metodología). Establezco que las novelas, además de su intertextualidad con nu- 
merosos referentes culturales, de su diálogo constante con varias tradiciones literarias y de su acendrado registro lingüístico, tienen el propósito central de trastocar el orden de lo Simbólico en que se han sostenido tradicionalmente grandes temas de la agenda mexicana e incluso latinoamericana a las cuales se refieren: narcotráfico, migración, violencia.

\title{
2. Construcción de metodología
}

\author{
precisamente por mirar al sesgo, \\ es decir, desde un costado, \\ ella ve la cosa en su forma clara y distinta \\ (Žižek, Mirando al sesgo, 2000: 29)
}

¿Cómo unos personajes marginales podrían trastocar el orden de lo Simbólico en que se sostiene su realidad y la idea que poseen de ella? Para responder a esta pregunta, diseñé una metodología que permite ahondar en los principales elementos de cada novela de la trilogía.

La primera parte de la metodología tiene que ver con lo que Žižek menciona en un video sobre cómo opera la mirada oblicua en Children of men. A decir del autor, la cámara no sigue solamente al personaje principal, sino que a cada instante se desvía y se queda fija en el trasfondo, donde hay una ciudad devastada. Esa es la mirada oblicua con que es posible contar no solo la historia que ocurre en primer plano sino, fundamentalmente, otra historia que, estando en el trasfondo, resulta temáticamente muy importante e incluso puede ser explicación de la historia aparentemente central: "[...] The true focus of the film is there, in the background [...] It's the paradox of what I would call anamorphosis. If you look at the thing to directly [...] you don't see it; you can see it in an oblique way only if it remains in the background [...]" (Žižek, 2014). La anamorfosis es el recurso que permite ver de manera correcta, sin deformaciones, un objeto.

Lo crucial es que entre la historia principal y la del fondo se establece una tensión importante, donde ambas se comunican y transforman mutuamente. En las novelas de la trilogía, los personajes de las historias en primer plano están en constante transformación debido al peso que tiene un lenguaje cambiante sobre ellos; en consecuencia, cuando miran hacia el trasfondo, éste sufre un cambio. Pero también ocurre la operación inversa: el trasfondo convoca la mirada de los personajes e incide en ellos. En suma, me interesa cómo la mirada oblicua crea un ámbito de tensión, en el cual se multiplican los significados de las historias que están en juego. 


\subsection{Resimbolización del narcotráfico, la migración, la violencia}

Lo relevante del recurso de la mirada oblicua radica en que el sujeto -en quien opera la anamorfosis mencionada por Žižek- mira hacia determinado tema y brinda al respecto una nueva interpretación. La pregunta es ¿qué nueva interpretación aportan las novelas? Para responder, hay que recurrir al planteamiento lacaniano de que el conocimiento que puede tener el sujeto está conformado simultáneamente por los órdenes de lo Real, lo Imaginario y lo Simbólico. El orden de lo Real se refiere a aquello que el sujeto nunca podrá conocer; es decir, se refiere a cómo lo Real es inasequible antes de que haga su aparición el lenguaje y lo simbolice, y cómo el sujeto solo puede conocer a través del lenguaje, ergo, lo Real es una imposibilidad para el sujeto. El orden de lo Imaginario sería el conjunto de articulaciones que el sujeto construye y que le permiten pensarse a sí mismo, relacionarse con los demás sujetos y vincularse con el orden de lo Simbólico. Y el orden de lo Simbólico, por último, es una articulación lógica, superior diríamos, del mundo por medio del lenguaje, y que siempre antecederá al sujeto, de modo que éste no puede sino sujetarse a ella. La unión de los tres es necesaria para que el sujeto comprenda el mundo (Lacan, 2009: 23-72).

En todo este entramado, lo primordial es el lenguaje. Lacan desarrolló el esquema "L", en el cual se explica que el sujeto es definido desde el primer momento de vida por el lenguaje que le impone el Otro (A) (Lacan, 2009: 62). El esquema "L" es la representación gráfica para designar la manera en que el Otro (A) - función que al comienzo de la vida desempeña la madre- determina al yo (a). La operación tiene lugar cuando el Otro le confiere al yo (a) el lenguaje, es decir el medio que le permite asimilar los órdenes Simbólico e Imaginario: gracias a esto queda diferenciado del Otro (A) y así, en suma, puede conocer el mundo.

Figura 1

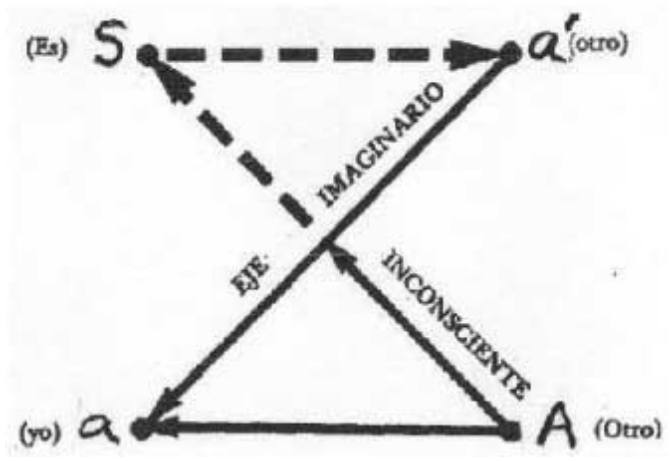

Esquema “L” 
Pero la operación del esquema "L" continúa. Después de conocer el mundo, el yo (a) puede reconocerse como otro (a'). Es importante aclarar que este otro (a' minúscula) es diferente al Otro (A mayúscula). $Y$ al reconocerse como un otro (a') distinto al Otro (A), el yo (a) entonces se asume como sujeto (S).

Ahora bien, un aspecto importante es que el sujeto (S) en ningún momento deja de estar en relación con el Otro (A). Es decir, el sujeto $(S)$ continúa determinado por ese primer momento en que el Otro (A) le otorgó al yo (a) el lenguaje. De tal suerte que el deseo del Otro (A) se inscribe en el deseo del sujeto (S). Dicho de otro modo, el sujeto (S) desea en tanto que el Otro (A) le ha inscrito su propio deseo. Toda esta condición hace que el sujeto $(S)$ sea más bien un sujeto partido, escindido, en él mismo y en el Otro (A) que, dicho de manera coloquial, le habita: por eso se dice que es un sujeto barrado, y se representa así: $\$$

El sujeto es delineado por el lenguaje desde un principio, y más tarde emprende un intenso proceso hacia la consolidación de su subjetividad. Este hecho, donde el lenguaje define al sujeto, es determinante en la operación de sujeción-subjetivación de los protagonistas de la trilogía fronteriza. De ahí la importancia de analizar cómo el lenguaje simboliza el mundo y se lo presenta al sujeto. Justamente la simbolización es la operación que más me interesa para la trilogía fronteriza, pues si el orden de lo Simbólico se sostiene en el lenguaje, es razonable pensar que la operación de la literatura, en tanto dispositivo artístico cuya materia prima es el lenguaje, pueda incidir en la resimbolización. En las obras de Herrera interesa explorar si hay algún trastrocamiento de ese orden de lo Simbólico, específicamente de la concepción que el sujeto tiene sobre el narcotráfico, la migración y la violencia, en el contexto mexicano contemporáneo en el que las novelas han sido escritas y ambientadas.

\subsection{Escritura sesgada}

El siguiente elemento importante de esta metodología es la mirada. Sin embargo, para entenderla hay que hablar antes del concepto de pulsión escópica, signado también por Lacan.

Destaco el papel que el fantasma desempeña conjuntamente con la mirada. La presencia del fantasma, en tanto presencia del Otro (A) en la constitución del deseo del sujeto, es muy importante para determinar la visión que tendrá el sujeto. Se puede reducir esto a una ecuación silogística. Si el deseo del sujeto está determinado por el Otro (A), y la visión del sujeto está determinada por el deseo, se pue- 
de afirmar que la visión del sujeto está determinada en gran medida por el Otro (A). Se entiende así la pulsión escópica como ese llamado que el objeto causa de deseo a produce en la mirada del sujeto; un llamado en el que está presente el deseo del Otro (A) inscrito en el sujeto. Entender que en la mirada está inscrito el deseo es muy importante para comprender la anamorfosis.

En el epígrafe de esta metodología (precisamente por mirar al sesgo, es decir, desde un costado, ella ve la cosa en su forma clara y distinta) Žižek (2014) está aludiendo a la anamorfosis. Es una operación mediante la cual una imagen, cuadro o pintura está aparentemente deforme. Es necesario que el observador cambie de posición y la mire desde un ángulo distinto, para que cobre su verdadera forma. Žižek precisa que si se mira con supuesta objetividad, lo único que hay es en el amorfo orden de lo Real, una mancha. Es necesario ver con una mirada alterada, interesada, 'distorsionada' por el deseo, para que la imagen tenga sentido.

Esta forma de operar de la mirada es central en mi planteamiento de lo que ocurre en las novelas: una escritura sesgada. No se puede hablar de mirada, en rigor, porque la materialidad de la literatura es el lenguaje. En este sentido, el concepto de escritura sesgada que planteo consiste en presentarnos personajes en primer plano cuya subjetividad se ve afectada por los objetos hacia los que observan, en el trasfondo, y que de regreso los miran a ellos: así los personajes sufren transformaciones al verse siendo vistos. Desde la posición tangencial que ocupan, los personajes observan lo que acontece en el plano de fondo, donde está la mancha amorfa de acontecimientos. Gracias a la mirada cargada de determinado deseo, debido al lenguaje que el Otro (A) ha inscrito en los personajes, todos esos hechos situados en el trasfondo adquieren sentido, al incorporarse en las historias de esos personajes.

\subsection{Discursos de poder}

Parto de un elemento muy importante en las novelas, el idioma español, para analizar si la mirada oblicua logra incidir en lo Simbólico. El núcleo de esta propuesta se encuentra en el planteamiento de "discurso" de Michel Foucault (1983) para referirse a las fuerzas de poder que atraviesan al lenguaje: "en toda sociedad la producción del discurso está a la vez controlada, seleccionada y redistribuida por un cierto número de procedimientos que tienen por función conjurar los poderes y peligros, dominar el acontecimiento aleatorio y esquivar su pesada y temible materialidad" (11). 
Debe entenderse el discurso como la intención del poder de imponer lo que Foucault daba en llamar "voluntad de verdad": el poder trata de imponer su "voluntad de verdad" como una verdad a secas (20). Ante lo cual, el propio Foucault habla de que hay que "poner en duda nuestra voluntad de verdad; restituir al discurso su carácter de acontecimiento; levantar finalmente la soberanía del significante" (43).

Lo importante es que ese lenguaje, en absoluto inocente, es el mismo que da forma al orden de lo Simbólico, esto es, a cualquier posible conocimiento sobre la realidad. Si el lenguaje en las novelas de Herrera comporta una intensa imbricación de diferentes niveles discursivos, se puede decir que este manejo del lenguaje se refleja en la construcción de un orden de lo Simbólico muy particular. La conciencia de cómo el lenguaje soporta determinados discursos de poder sobre el narcotráfico, la migración y la violencia en México es nítido en la trilogía; de suerte que, a través de los muchos carices que éstas adoptan a nivel del lenguaje, le plantean al sujeto nuevas interpretaciones sobre esos temas. La operación consistente en identificar los discursos de poder que subyacen a un gran tópico es un vaso comunicante en la trilogía fronteriza. Y es una operación que se explica a través de la propuesta de Foucault.

\subsection{Sujeto, sujeción y subjetividad}

En el centro de esta metodología está la constitución del sujeto, entendido a partir de Lacan (2009), quien explicaba que su programa pasaba por indagar "cómo un lenguaje formal determina al sujeto" (52). Pero, como el mismo Lacan aclara, "semejante programa no es simple: puesto que supone que un sujeto no lo cumplirá sino poniendo algo de su parte" (52). En efecto, si el sujeto en primera instancia depende de la predominancia del Otro (A), llega un momento en que debe "poner de su parte" para adquirir el rango de sujeto y subjetivarse.

La operación que plantea Lacan y que el sujeto debe seguir hacia su subjetivación es la siguiente. Ya he referido que el Otro (A) incompleto, es la función que brinda el lenguaje al sujeto, y por lo tanto, en un primer momento, lo determina, tal como explico en el esquema "L". Esto tiene una relación importante con las novelas de Herrera, porque en ellas, de diferentes formas y en diferentes grados, ocurren constantemente movimientos de sujeción y subjetividad, conceptos trabajados también por Žižek y Judith Butler.

Pero, ¿por qué ocupa el centro de esta metodología el análisis del sujeto? Porque las novelas muestran una gran conciencia de las ma- 
neras en que presentan a los sujetos y las circunstancias que los determinan, o sea que los subjetivan, tales como la relación con el Otro (A), las condiciones del lenguaje, la intersubjetividad con otros sujetos, etcétera.

Butler (1997) precisa que "aunque el sujeto ingresa en la normatividad del lenguaje, el sujeto solo existe como una ficción gramatical previa a dicha entrada. [...] las normas que rigen la formación del sujeto parlante separan al sujeto de lo inexpresable, esto es, producen lo inexpresable como la condición de la formación del sujeto" (222). Ocurre una operación doble: el sujeto adquiere subjetividad, al tiempo que se sujeta, atado, a un lenguaje del que no puede sustraerse.

La cuestión se precisa aún más con Žižek (2010), cuando habla de la forma en que el sujeto, al realizar un acto de habla performativo, el que habla no es el sujeto sino el Otro $(\mathrm{A})$ a través suyo: "en el gesto mismo de lograr un acto mediante la pronunciación de palabras, soy privado de la autoría, el 'gran Otro' (la institución simbólica) habla a través de mí" (133). La importancia de pensar el lenguaje en estos términos, es que en la trilogía el lenguaje define las estructuras más internas del sujeto.

La subjetivación/sujeción ocupa el lugar central de esta metodología porque es necesario analizar qué ocurre con el sujeto frente al trastrocamiento del orden de lo Simbólico. De tal forma, se analiza la subjetivación de cada uno de los protagonistas, y en consecuencia, la construcción de nuevos órdenes de lo Simbólico que re interpreten el narcotráfico, la migración y la violencia.

\section{Subjetividades que cuestionan}

\subsection{Señales... del devenir}

Al explicar lo que ocurre con la imagen que el sujeto tiene de sí mismo, los psicoanalistas argentinos Eduardo Carbajal, Rinty D’Angelo y Alberto Marchilli (2006) señalan que "la imagen en la que el sujeto se aliena implica no solo la imagen que ve efectivamente en el espejo sino fundamentalmente las significaciones producidas por el $A^{\prime \prime}$ (p. 100). Esto es muy importante en Makina y en la transformación que experimenta a lo largo de su viaje. Si ya desde el comienzo ella hablaba tres lenguas y, en consecuencia, estaba sujeta a un Otro (A) complejo, debido al influjo de esas lenguas en su subjetividad, cuando más tarde tiene lugar su transformación y ella se mira de otra manera, la forma en que ella se ve estará influida por ese nuevo Otro (A) que está incidiendo en ella. 
Se puede notar esto si se comparan dos fragmentos de la novela. Al comienzo, Makina se encuentra en un pasillo con espejos a ambos lados, entonces "miró dentro de los espejos: enfrente de ella estaba su atrás: ella miró a través, pero encontró solo un frente sin fin, trascurvita, como invitándola a seguir a través de los umbrales" (2011: 22). De este fragmento quiero destacar que la imagen que Makina tiene de sí misma es harto compleja. La frase "enfrente de ella estaba su atrás" puede representar, de acuerdo con los psicoanalistas argentinos, que Makina está sujeta a un Otro (A) inestable, inextricable.

En contraste, poco antes de terminar la novela, Makina se encuentra con el grupo de personas migrantes postradas de hinojos ante un policía que las humilla, y le arrebata al policía el cuaderno que a su vez le había arrancado a una de esas personas: "Nosotros somos los culpables de esta destrucción, los que no hablamos su lengua ni sabemos estar en silencio [...] Los que quién sabe qué aguardamos. Nosotros los oscuros, los chaparros, los grasientos, los mustios, los obesos, los anémicos. Nosotros, los bárbaros" (2011: 99-100).

Cuando Makina escribe esto, es ya un sujeto que se identifica plenamente con esas personas que estaban siendo humilladas. A diferencia de la Makina del comienzo, cuando el Otro (A) que definía la imagen que ella misma vio en el espejo era otro (A) complejo, debido a las tres lenguas que ella hablaba, aquí, al final de la novela, ella - sujeto en devenir, como lo he propuesto- se identifica con la imagen de esas personas siendo humilladas. Es decir, su proceso de transformación que no cesa, como ha propuesto Ivonne Sánchez Becerril, se refleja en el proceso que viven los paisanos: es uno de los puntos de diálogo entre la historia de Makina en primer plano y la del trasfondo, de las personas migrantes. Sánchez Becerril (2014) ha afirmado que Señales... refleja el elemento de inestabilidad permanente que se inscribe en el sujeto migrante: "El análisis de la mirada de soslayo que hace Señales... de la movilidad internacional pone en relieve la complejidad e impacto del fenómeno de largo plazo en la transformación social tanto de México como de Estados Unidos" (108).

La mirada, y el modo en que la emplea Makina, es muy importante en la transformación que planteo; en particular, porque esa transformación no es solo un aprendizaje, sino una resubjetivación: hay un cambio de autoridad, una presencia de un Otro (A) distinto, en la subjetivación de Makina. ¿Cuál es el cambio que opera en Makina? Una respuesta la encontramos en el planteamiento de Santiago Navarro (2011): "Yuri Herrera [...] apunta al carácter caduco de la propia categoría de identidad, o al menos de la obcecación identitaria 
esencialista. En sus representaciones de la experiencia del migrante se destacan [...] facetas más bien fecundas como la del dinamismo (en sentido amplio) [...]" (117).

La noción de dinamismo es adecuada para dirigirse hacia el tipo de Otro (A) que sostiene la subjetividad de Makina, durante y después de los episodios en que experimenta lo que llamo devenir: expresión con la cual señalo que en Makina no se trata de una transformación que la conduce hacia un punto final, sino que esa transformación se trata de un proceso permanente, incesante.

Y esta transformación es patente por la mirada, pero igual lo es por el lenguaje, a través del cual el sujeto comparece en varios momentos. Quizá uno de los más notables sea cuando Makina camina con su hermano, y éste se encuentra con un soldado amigo suyo:

Ellos se encontraron con un soldado golpeado que comenzó a hablar con el hermano de Makina:

Anoche iré a un bar al que nos dirán, le dijo en gabacho.

Ah, ¿sí? Y qué tal, gabacheó su hermano (2011: 91-92).

Su hermano le explica a Makina que ese soldado está practicando su inglés, y ese día había tocado en futuro. Interpreto lo que ocurre allí como el proceso de transformación de Makina. Ese muchacho que habla español, pero practica su inglés contando lo que ocurrió la noche anterior en tiempo verbal futuro es la muestra fehaciente de ese permanente cambio de Makina: apropiación del pasado desde el presente en términos de futuro, en un idioma que se está apre(he)ndiendo.

En suma, el Otro (A) muy complejo al que se sujeta Makina al final de la novela es uno distinto al del principio. Y desde esa complejidad mira hacia la migración: la entiende como irreductible a categorías firmes. Y las personas migrantes, alejadas de las identidades rígidas, se vuelven hacia una permanente transformación. Este es el orden de lo Simbólico que plantea la novela sobre la migración contemporánea México-Estados Unidos. Es al mismo tiempo una novela fronteriza porque Makina y los personajes están siempre en esa condición. Su forma de ver el mundo está en el umbral del dinamismo identitario. Y en ese proceso, el descenso al Mictlán es una ratificación; por eso, cuando Makina llega a la cámara sin ventanas ni orificios para el humo, es cuando se instala, de forma definitiva, en esa inestabilidad. 


\subsection{Trabajos del orden de lo Simbólico}

El propio Herrera ha hablado de su objetivo al construir Trabajos del reino:

Si uno habla ya de narcotráfico, de capo, de narcocorridos, está reproduciendo una serie de clichés con los cuales nos bombardean la propaganda gubernamental y los medios masivos de comunicación, y parte de la responsabilidad de la literatura es encontrar un nuevo lenguaje para lo que nos está sucediendo y para lo que nosotros estamos construyendo (Herrera, 2012).

Frente a esto, sin embargo, Oswaldo Zavala ha postulado que Trabajos del reino es acrítica respecto a la realidad a la que se refiere; ha argumentado que lejos de cumplir su cometido, la novela reproduce de manera acrítica el discurso gubernamental.

Es decir, esta novela, para Zavala (2014), "displays the same ethical impasse found in most narconarratives by reproducing the simulacrum of truth about drug cartels promoted by official discourse" $(346)^{4}$. En el mismo texto, concluye que:

[...] most of the narconarratives written during the last decade in Mexico reify the simulacrum of truth constructed by official propaganda [...], critical narconarratives must abandon the exhausted myths of drug lords and their fantastic kingdoms and stop objectifying drug trafficking as a problem external to official power in Mexico and the U.S. and instead propose a careful historical revision of its place inside that power: drug trafficking as power itself (357) .

Ciertamente, Trabajos del reino parecería tramarse partiendo de una posición fácilmente reconocible: la de los "discursos oficiales", ya sea los difundidos por el gobierno o por los medios de comunicación so-

4 "Muestra el mismo estancamiento ético que se encuentra en la mayoría de las narconarrativas, al reproducir el simulacro de la verdad sobre los cárteles de la droga reproducido por el discurso oficial" (trad. mía).

5 [...] la mayoría de la narconarrativa escrita durante la última década en México reifica el simulacro de la verdad construida por la propaganda oficial [...] la narconarrativa crítica debe abandonar los gastados mitos sobre los capos de la droga y sus fantásticos reinos, y dejar de objetivar el narcotráfico como un problema externo al poder oficial en México y en EEUU, y proponer una cuidadosa revisión histórica de su lugar dentro del poder: el narcotráfico como el poder mismo (trad. mía). 
bre el narcotráfico en México, que menciona Zavala, y algunos de los cuales se revisaron en la sección anterior.

Pero sugiero que esa crítica de un ámbito exterior y otro interior, en los términos en los que ha planteado el tema Luis Astorga (2007) y que retoma el propio Zavala, es la misma que sugiere la novela. El análisis que presento a continuación postula cómo, aun cuando al comienzo la historia de Lobo parece tramarse a partir de discursos conocidos sobre el narcotráfico mexicano de las últimas décadas, e incluso pareciera que los estetiza al presentarnos a un Lobo que se maravilla con lo que encuentra en el Rey y el Reino, lo cierto es que a medida que avanza la historia nos damos cuenta de que estamos asistiendo más bien a una historia que plantea desmontar esos discursos. Es decir, si los presenta es con el fin de cuestionarlos.

La primera operación que tiene lugar en la novela se debe al recurso formal de singularización o extrañamiento, que, tal como lo plantea Shklovski, consiste en nombrar las cosas no por su nombre conocido sino con términos próximos. Se presentan las cosas de forma un tanto extraña. Que se emplee este recurso en la novela es muestra de la conciencia de los discursos de poder y de la materialidad que encierra el lenguaje. Si no se utilizan términos como narcotráfico, capo, cartel, drogas u otros semejantes, es porque se busca una distancia de los discursos hegemónicos que sobre el narcotráfico poseen tales términos.

En este sentido, lo importante es la operación siguiente de la novela, consistente en la transformación de mirada oblicua plena de deseo, en términos lacanianos. Desde la posición tangencial que ocupa Lobo, y que en todo momento lo mantiene alejado de las actividades que ocurren en el Reino, se maravilla al principio, pero a medida que su visión se aclara y su entendimiento se abre — gracias a los anteojos que da el personaje del Doctor y a los libros que le obsequia el Periodista-, desde esa misma posición lateral y distante consigue ver hacia el centro con mucha más precisión. Es decir, el recurso de singularización, que al comienzo sirvió para estetizar lo que ocurría en el Reino, impedirle a Lobo entender lo que ocurre y entonces maravillarse, resultó haber sido una condición necesaria para la operación final: develar que no hay ningún misterio en lo que ocurre dentro del Reino. Todo el tiempo fue un espectador deslumbrado por el faro de luz que es el Rey, y su posición era distante. Desde esa posición lateral ocurre la mutación de su pulsión escópica y, en consecuencia, de su mirada. El resultado es que ahora observa de manera muy diferente lo que ocurre allí: comprende que no hay nada de maravilloso sino que, por el contrario, se trata de un ámbito mundanamente criminal. Esta sería, desde mi análisis, la reinterpretación que la novela ofrece sobre el orden Simbólico del narcotráfico. 


\subsection{Transmigración del miedo y la violencia}

El Alfaqueque, protagonista de la tercera novela, está inspirado en los alfaqueques mencionados en las Partidas de Alfonso X, "El Sabio", un documento normativo del siglo XIII. Eran redentores de cautivos que debían cumplir, entre otras, con las siguientes condiciones: "que sean sabedores tanto del lenguaje de aquella tierra a la que van, como de la suya" (65), y que sepan "conservar el secreto, pues si tales no fuesen, no podrían guardar su verdad" (65). Que en la novela el Alfaqueque cumpla con estas características es esencial pues en el "reino del Verbo" (Herrera, 2013: 61), donde tiene lugar su historia, una de sus funciones básicas es "mermar verdades de piedra" (99), para lo cual tiene que echar mano magistralmente de su arma principal: el lenguaje.

Lo primero que aquí analizaré es de qué manera se constituye el deseo en el Alfaqueque. A partir de Lacan, convenimos en que el lenguaje es otorgado por la autoridad del Otro (A) al sujeto. Lo interesante es que también le transfiere su deseo. De modo que el deseo que posee el sujeto es una transmisión del deseo del Otro (A). Y si las principales manifestaciones del deseo son las pulsiones, en este caso, la pulsión escópica, resulta que lo que el sujeto desea ver es en cierta medida lo que el Otro (A) le ha transmitido.

En La transmigración de los cuerpos, al revés de lo que ocurre en las otras dos novelas, no hay un cambio de la autoridad representada por el Otro (A). Mientras que en aquellas dos, los protagonistas se separan de una sujeción inicial y abrazan otra, muy diferente, en esta tercera novela el Otro (A) se mantiene invariable. Esto es importante para analizar la forma en que el sujeto mira, desde la posición que ocupa su historia en primer plano, hacia la violencia y el miedo situados en el trasfondo de la historia.

La historia que ocurre en primer plano es la del Alfaqueque, quien debe resolver, echando mano de su dominio del lenguaje, la entrega de los cuerpos de La Muñe Castro y Romeo Fonseca a sus respectivas familias, y desde esa posición mira hacia el trasfondo. A través de su mirada, definida por el deseo transferido por un lenguaje complejo cuya principal característica es su carácter utilitario, mira hacia el trasfondo donde hay una mezcla de desconfianza, inseguridad, temor.

Ahora bien, la violencia y el miedo dominantes del trasfondo han logrado filtrarse en la historia del Alfaqueque, de forma que no solo es testigo presencial de los episodios violentos, sino quetambién su historia es resultado de esa violencia. Está el ejemplo de cuando, manejando su carro, se encuentra con un retén militar que lo detiene. El soldado de pronto deja de verlo a los ojos y dirige la mirada hacia un 
muchacho que había sido detenido: “[...] se volvió hacia los soldados junto al camión y dijo Quítenle esas pendejadas. Uno de los soldados maniató por detrás al muchacho y el otro empezó a arrancarle los aretes. El punketo se revolvía en silencio, hilillos de sangre comenzaron a escurrirle de las cejas, de la nariz, de la boca" (Herrera, 2013: 56). El miedo y la violencia se encuentran tanto en el trasfondo como en el plano que ocupa el Alfaqueque. Similar es este ejemplo: "Vio por el retrovisor una camioneta negra que se le atrabancaba unos metros atrás [...] De ella bajaron dos hijuelachingadas con caras de ser muy hijuelachingadas. El que bajó del lado del pasajero no necesitó ordenarle al Alfaqueque que se acercara. El Alfaqueque apagó el coche y bajó" (99-100).

Lo que ocurre es que el Alfaqueque mira hacia esos hechos a través de su mirada plena de un deseo determinado por un lenguaje utilitario y advenedizo, cuya característica es servir de herramienta para mediar conflictos y obtener favores sexuales. El sujeto se asume únicamente como el lenguaje que posee, o bien, como instrumento de ese lenguaje. El Alfaqueque, cuya historia ocurre en un primer plano lleno de violencia y miedo, mira hacia el trasfondo donde igual reinan la violencia y el miedo. Es, pues, un mero reflejo del trasfondo.

Entonces, en la historia del Alfaqueque, ¿ocurre una resimbolización del gran tema hacia el que mira oblicuamente? Durante toda la historia, el sujeto no transforma su tipo de sujeción al lenguaje, por más complejo y rico que éste sea: permanece sin variaciones de principio a fin la figura de autoridad representada por un Otro (A) cifrado en el lenguaje que sujeta al Alfaqueque. De tal modo que la mirada del Alfaqueque parece ser menos oblicua que directa: resulta que no se trata de dos planos en tensión, sino que en ambos hay violencia, miedo, desconfianza.

Esto se traduce en que la operación que ocurre en La transmigración... es la menor de la trilogía. En la consciencia del Alfaqueque, a propósito de su rol como un instrumento del lenguaje, se encuentra una de las principales metáforas de la novela: la de que personajes como él - que cifran su valía en un lenguaje que les permite sortear las inseguridades, mentiras, injusticias, etcétera, del medio ambiente en que se encuentran - son un tipo de subjetividad generada por el proceso de modernidad desigual en Latinoamérica. Pero el hecho de que el Alfaqueque no sufra una transformación y de que su historia se limite a ser de inicio a fin consecuencia directa de esa violencia hacia la que mira, hace de esta novela un ejercicio literario de menor alcance que las otras dos, aun cuando el reto que se planteaba (utilizar el miedo como metáfora del medioambiente latinoamericano) es interesante y aun urgente en Latinoamérica. 


\section{Conclusiones. Sujeto, sujeción, subjetivación}

La idea de que a lo largo de Trabajos... y de Señales... sí hay una transformación del orden de lo Simbólico mientras que a lo largo de $L a$ transmigración ... no, está estrechamente ligada con lo que analizo en esta última sección.

\subsection{Makina, la sujeción a un distinto Otro (A)}

Margarita Ramón-Raillard (2013) ha seguido el viaje emprendido por Makina, deteniéndose en numerosos elementos que le permiten afirmar que, más que tratarse de una frontera física, lo destacable de la novela es la pertinacia de 'lo fronterizo' que se expresa básicamente en la forma de entenderlos por parte de Makina y de las personas migrantes, en la forma en que Makina mira y va cambiando su forma de pensar; en el nuevo idioma en constante transformación, el cual es expresión directa de su propia condición de devenir. Se explicaría esto como una especie de estado donde lo fronterizo atraviesa al sujeto. Me refiero a la operación de la que ya he hablado: en Makina, a medida que se adentra en ese espacio que es a un tiempo territorio estadounidense y Mictlán, las tres lenguas que domina desde el comienzo, actúan en dos sentidos. Por un lado, le permiten entender que tiene frente a sí una nueva lengua, pero a la vez deja de estar sujeta y subjetivada por tales lenguas, y comienza a estarlo con respecto a esa nueva. Ahora se enfrenta a una lengua mixturada, se instala en un estado de devenir, y lo que acaba por subjetivarla es la inestabilidad.

Santiago Navarro (2011) emplea el término "flexibilización" para analizar lo que ocurre con Makina. Huye, como yo, de la categoría de "identidad". Él precisa cómo actúan los hechos que Makina observa en los migrantes, para la transformación que obra en su interior: "Las transformaciones que experimenta el lenguaje de los migrantes actúan como correlato objetivo de sus mutaciones identitarias" (119).

Idea que suscribo: las transformaciones que Makina observa en sus paisanos - comportamientos, formas de hablar, de convivir...- la transforman a ella misma, si bien, sostengo que los cambios que Makina nota en el lenguaje son los que más inciden en su propia mutación.

Este planteamiento cobra un valor especial cuando se liga con lo que afirma Gloria Anzaldúa (2016), quien lleva esta operación a un punto concreto: la frontera entre México y Estados Unidos, que conceptualmente es donde yo ubico los cambios que experimenta Makina y los migrantes mismos (105-106). Me valgo de este planteamiento 
de Anzaldúa para apuntalar el proceso de transformación por el que atraviesa Makina: un proceso que es resultado del cruce de identidades contenidas en los idiomas que se mezclan en el Gabacho, y cuya fusión Makina puede observar porque ese mismo cruce está ocurriendo en ella y en las tres lenguas que domina.

De los planteamientos de Navarro, Ramón-Raillard y Anzaldúa, desprendo que Makina es una metáfora del tránsito; que se trata de un personaje atravesado por los cambios que está sufriendo su propio lenguaje y, en consecuencia, su manera de enunciar el mundo es igual diferente. Faltaría agregar otro resultado de esta transformación: la forma en que mira al mundo — que también está atravesada por ese cambio.

Me gustaría llevar estos planteamientos al terreno de lo que planteé desde el comienzo, en los términos de sujeción/subjetividad desde Lacan hasta Butler y Žižek. El sujeto solo puede existir "en tanto que forma parte del lenguaje" (Butler, 1997: 222). Por su parte, Lacan (2009) hablaba de "cómo un lenguaje formal determina al sujeto" (52), esto es, una formación inicial de la subjetividad que más adelante el sujeto debe completar, poniendo de su parte.

¿Qué sucede cuando cambia en Makina su dominio de las tres lenguas? Al mismo tiempo que deja de estar sujetada a las reglas de los tres idiomas, pierde cierta subjetividad; sin embargo, gana la posibilidad de participar de la comunicación con otros sujetos que viven la nueva lengua. No hay que olvidar lo que precisa Lacan a propósito de los órdenes de lo Real, lo Imaginario y lo Simbólico: lo Real no sería más que una mancha incomprensible, como la llama Žižek, si no acudiera el lenguaje en busca de simbolizarla, esto es, de darle sentido, de ofrecer comprensión. La nueva lengua, que sujeta y subjetiviza a Makina, es la que acude a dar sentido a ese proceso fronterizo, migratorio, que observa ella.

Makina dejó de ser el sujeto sujetado a las tres lenguas y al deseo del Otro (A) que se manifestaba a través de esas lenguas. Con la operación de ganancia de una lengua nueva, en permanente cambio, pasó a ser un sujeto sujetado a Otro (A) que aquí he dado en llamar "devenir". Si lo que hace el lenguaje es manifestar en el sujeto el deseo permanente del Otro (A) por ir en busca de lo Real y tratar de simbolizarlo, lo que ocurre con una Makina sujetada a un Otro (A) que es puro devenir, cuyo lenguaje es pura transformación, es que para ella todo lo Real es transformación. De tal modo, esa mancha de la que habla Žižek se difumina mediante el lenguaje cambiante que la apresa: el mismo lenguaje que ahora sujeta a Makina. 
Esto se traslada concretamente a la interpretación que se pudiera ofrecer desde la novela sobre la migración México-Estados Unidos. De hecho, es posible por lo que ocurre con la mirada oblicua: como hay, dicho esquemáticamente, una Makina que se transforma, entonces su mirada se transforma, y, como consecuencia lógica, lo que mira también se transforma. Lo mismo que le ocurre a ella parece ocurrirles a las personas migrantes que se sitúan dentro de una identidad en permanente transformación, gracias a la cual se logran adaptar en cada momento a las condiciones que les impone el contexto.

\subsection{Lobo, recuperar el nombre}

En el caso de Trabajos del reino, a la vez que Lobo/Artista se apropia del lenguaje a través de los libros que le proporciona el Periodista (quien le había hecho ver que "si uno disfruta las palabras es como pistear con el oído" [Herrera, 2010: 36]), se sujeta cada vez más a ese lenguaje lleno de reglas, de normas, de historia, de una carga como la que menciono antes, plena de materialidad. Butler (1997) señalaría que "si un sujeto deviene sujeto al entrar en la normatividad del lenguaje, entonces esas reglas preceden y determinan la formación misma del sujeto de forma significativa" (222).

Ahora bien, el proceso de subjetivación que describe Butler tiene lugar gracias a la presencia de un Otro (A). Ese Otro (A) es una autoridad que también cambia a medida que cambia la forma en que Lobo/Artista se relaciona con el lenguaje. Para analizar cómo se transforma la sujeción de Lobo/Artista por la presencia de ese Otro (A) cambiante, me baso en una propuesta žižekiana a propósito de la subjetivación, que menciono desde la metodología. Al hablar de la ópera Carmen de Bizet, Žižek (2000) ha dicho que Carmen, aun cuando creía que "manejaba los hilos", en realidad era un objeto para los hombres: hasta que advierte este hecho, se "subjetiviza", o sea, se convierte en sujeto (111).

¿Por qué pasa esto? Explica Žižek (2000) que "la subjetivización es estrictamente correlativa del hecho de experimentarse a uno mismo como un objeto [...]" (111). Pues bien, eso le ocurre a Lobo. Lo cual queda de manifiesto cuando habiendo escrito un corrido en loor del Rey, éste lo increpa y le dice el lugar que ocupa en el Reino:

- Señor, yo pensé...

— ¿De dónde sacaste que podías pensar? ¿De dónde?

Tú eres un soplido, una puta caja de música, una cosa que se rompe y ya, pendejo (Herrera, 2010:109). 
Queda patente el lugar que ocupa en el Reino: el de un músico que debe cantar elogios a su jefe. Entonces, al tiempo que adquiere conocimiento del lenguaje y de la palabra, Lobo adquiere también la subjetividad que le permitirá ver lo que ocurre en el Reino. Varía allí la forma en que el sujeto comprende el orden de lo Simbólico del narcotráfico: lo que al comienzo le parecía una certeza sobre la imagen casi heroica del Rey, más tarde se transforma en la certeza de que él no es un héroe sino, como se lee en una cita ya mencionada: "un pobre tipo traicionado. Una gota en un mar de hombres con historias" (118). El sujeto constituye una nueva comprensión del orden de lo Simbólico del narcotráfico, a partir de que él mismo se transforma en sujeto consciente, y deja de ser un objeto al que los saberes llegaban acríticamente.

La figura de autoridad del Otro (A) que rige al Lobo del final de la novela, es una que le hace ver a la sociedad, al gobierno y, sobre todo, al narcotráfico como ámbitos compuestos por sujetos iguales a él, no hay superioridades ni inferioridades de ningún tipo sino "un hombre sin poderes sobre la tersa fábrica en la cabeza del Artista" (118). La figura de autoridad del Otro (A) en Lobo, dejan de cumplirla el Rey y su deseo. Ahora su lugar lo ocupa un Otro (A) anclado en el lenguaje-conocimiento y en los lentes-claridad de miras que le permiten ver las cosas sin más filtro de deseo que, aparentemente, el del saber. Y en consecuencia, el Rey y el Reino son despojados de ese misterio que aparentemente le eran propios. Quedan reducidos a un cártel más, encabezado por "un pobre tipo traicionado".

Vista así, pienso que la novela verdaderamente logra un trastrocamiento en los discursos de poder a propósito del narcotráfico, pues la imagen inicial del Rey y el Reino sufre una profunda transformación, y al final la imagen es una muy diferente. Cambia la forma de entender los discursos que se han utilizado repetidamente para cierta simbolización del narcotráfico. El mecanismo que sigue la novela es el que el propio Foucault sugiere para poner en duda la voluntad de verdad de los discursos dominantes; es un mecanismo que parte de la palabra, para ir hacia la resimbolización del modo en que el sujeto concibe la realidad.

En el paso del capítulo 25 al 26, es decir, del penúltimo al último, el Artista recupera su nombre, Lobo. Es una patente de que lo importante en la transformación de los discursos y del orden de lo Simbólico del narcotráfico ocurre en el seno del sujeto. Al recuperar el nombre que poseía antes de ingresar al Reino y de ser nombrado Artista por el designio del Rey, no solo renuncia al poder que el Rey en tanto Otro (A) ejerce sobre él; tampoco vuelve a ser nada más el mismo que al comienzo, sino que es nuevamente el sujeto que era 
pero habiendo transformado su subjetividad. No es pues en otro lugar sino en el sujeto y en la transformación de su subjetividad donde se desarrolla el centro de la propuesta de la novela de reinterpretar el narcotráfico.

\subsection{Alfaqueque, objeto subjetivado}

Para concluir este análisis, se podría decir que en La Transmigración de los Cuerpos no atestiguamos cómo se construye ningún sujeto ni ninguna subjetividad, sino que desde el arranque de la historia funcionan la propuesta del tipo de sujeto y subjetividad que se plantean. No hay, en suma, resubjetivación.

Una cita ("con el tiempo descubrió que lo suyo era navegar con bandera de pendejo y luego sacar labia. Verbo y verga, verbo y verga, qué no" (Herrera. 2013: 21)) está casi al principio. Es decir, desde un inicio se ilustra que el lenguaje, entendido como la herramienta que servía a los alfaqueques descritos en las Partidas de Alfonso "El Sabio", es el pilar en la constitución del sujeto y de la subjetividad que planteados.

La propuesta de la novela es ese lenguaje del Otro (A) que determina al sujeto, y que se expresa en fragmentos como "ayudaba al que se dejaba ayudar [...] y para eso es que servía ajustar el verbo" (49). Es un lenguaje que tiene una función definitoria para un sujeto como el Alfaqueque, en quien el trabajo con el lenguaje es, más que su medio de conocer el mundo como plantea Lacan, su forma de vida consciente. El tipo de sujeto y de subjetividad que propone la novela sería uno en el que se hace necesario el uso del lenguaje conscientemente a favor de la solución de conflictos, sin poner de por medio las opiniones personales. Y es imposible separar tal propuesta de la novela del tema central hacia el que mira el Alfaqueque: la violencia y el miedo social que rodean a las sociedades en Latinoamérica.

Ahora bien, dado que planteo que el sujeto en el transcurrir de la novela no se transforma, la consecuencia es que tampoco se trastoca el orden de lo Simbólico, sino que se mantiene inalterado.

Así es que frente a las dos novelas previas, que nos presentan la construcción de una determinada subjetividad, en esta no se hace más que verificar en la historia del Alfaqueque el orden de lo Simbólico construido a partir de la serie de hechos de los que hablaba antes: la violencia, la epidemia, el miedo, etc.

En todo caso, el Alfaqueque es una especie de correa de transmisión que permite que los odios almacenados, la violencia, los deseos de venganza, se mantengan latentes sin explotar. Tal me parece la pro- 
puesta de la novela en un tipo de sujeto como el que se presenta desde el comienzo.

\section{Bibliografía}

Alfonso X. Las siete partidas. Revisado el 7 de ago. de 2018: http://ficus. pntic.mec.es/jals0026/documentos/textos/7partidas.pdf

Anzaldúa, G. (2016). Borderlands / La Frontera: La Nueva Mestiza. (1 ${ }^{a}$ Ed.). España: Capitán Swing Libros S. L.

Astorga, L. (2007). Seguridad, traficantes y militares: el poder y la sombra. (1르. Ed.). Ciudad de México: Tusquets.

Butler, J. (1997). Lenguaje, poder e identidad. (1 ${ }^{\mathrm{a}}$ Ed.). España: Editorial Síntesis.

D’Angelo, R. et al. (2006). Una introducción a Lacan. (9ª Ed.). Buenos Aires: Lugar editorial.

Foucault, Michel. (1983). El orden del discurso. (2 ${ }^{\underline{a}}$ Ed.) Ciudad de México: Tusquets Editores.

Herrera, Y. (2012) . Apuntes para la manufactura Yuri Herrera en el MAZ. Revisado el 7 de agosto de 2017 desde Internet: https://youtu.be/Q8D-mLI-Zyk

. (2016). Eight questions to Yuri Herrera. 3:AM MAGAZINE. Revisado el 7 de agosto de 2017 desde Internet: http:// www.3ammagazine.com/3am/eight-questions-for-yuriherrera/

. (2010). Trabajos del reino. (3ª ed.) Barcelona: Periférica.

. (2011). Señales que precederán al fin del mundo. (2ª Ed.). Barcelona: Periférica.

. (2013). La transmigración de los cuerpos. (1를.). Barcelona: Periférica.

Lacan, J. (2009) Escritos 1. (3ª Ed.). México: Siglo XXI Editores.

Lombardo, M. Autoridad, transgresión y frontera (sobre la narrativa de Yuri Herrera). INTI, Revista de literatura hispánica, № 7980, 2014, 193-214.

Navarro Pastor, S. (2011). La violencia en sordina en Señales que precederán al fin del mundo de Yuri Herrera. iMex. México Interdisciplinario. Interdisciplinary iMex, I, 93-126.

Ramón-Raillard, M. Miradas cruzadas sobre la frontera MéxicoEstados Unidos a través de la narrativa mexicana del nuevo 
milenio: David Toscana (El ejército iluminado, 2006), y Yuri Herrera (Trabajos del reino, 2004 y Señales que precederán al fin del mundo, 2011). Revue de L'Institut des langues et cultures d'Europe, Amérique, Afrique, Asie et Australie, 18, 2013, 1-25.

Rodríguez Court, E. (2013). La precisión de la palabra: 'La transmigración de los cuerpos' de Yuri Herrera. Revista de letras. Revisado el 7 agosto de 2017 desde Internet: http://revistadeletras.net/la-precision-de-la-palabra-latransmigracion-de-los-cuerpos-de-yuri-herrera/

Sánchez Becerril, I. (2014). México nómada: Señales que prcederán al fin del mundo de Yuri Herrera, y Efectos secundarios de Rosa Beltrán. En Silvana Serafín. Escrituras plurales: Migraciones en espacios y tiempos literarios. (pp. 107-121). Milán: La Toletta.

Zavala, O. (2014). Imagining the U.S.-Mexico Drug War: The Critical Limits of Narconarratives. Comparative literature. 66:3, 340360.

Žižek, S. (2014). Children of Men: Comments by Slavoj Žižek Grym. Entrevista en video revisada el 7 de agosto de 2017 desde Internet: https://youtu.be/yqlqVcCPRd0 . (2010). El acoso de las fantasías. (1a Ed.). Ciudad de México: Siglo XXI. . (2000). Mirando al sesgo: Una introducción a Jacques Lacan a través de la cultura popular. (3ª Ed.). Madrid: Paidós. 